\title{
Relação entre presenteísmo, síndrome de burnout e liderança ética em organizações escolares
}

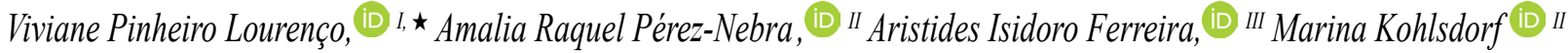 \\ ${ }^{I}$ Faculdade Laboro, Brasília, DF, Brasil

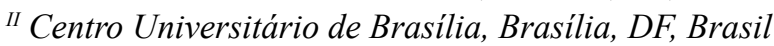

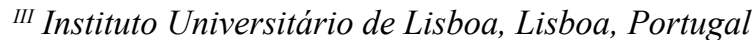

\section{Resumo}

O objetivo deste trabalho foi descrever variáveis preditoras de presenteísmo no contexto escolar, especificamente o burnout e a liderança. Utilizaram-se questionários como instrumento de coleta de dados. Responderam aos questionários 366 professores vinculados à Secretaria de Estado de Educação do Distrito Federal. Eles trabalhavam em diferentes escolas de várias regiões do Distrito Federal. Foram testadas relações lineares e com interação. Os resultados obtidos mostraram que a exaustão emocional e a liderança ética estão relacionadas à concentração no trabalho. Contudo, não se relacionam com a perda de capacidade para completar o trabalho. Neste último, a liderança aparece como uma importante variável moderadora. Os altos níveis de exaustão emocional tendem a convergir negativamente em avaliações de liderança e presenteísmo. Este estudo contribui para a compreensão da relação entre bem-estar e produtividade e suas variáveis moderadoras, assim como aponta que a exaustão emocional no trabalho influencia a avaliação do professor.

Palavras-chave: presenteísmo; síndrome de burnout; liderança ética.

\section{The relation between presenteism, burnout syndrome and ethic leadership in scholar's organizations}

\begin{abstract}
This paper aimed to describe predictors of presenteism in the school context, specifically burnout and leadership. Questionnaires were used as a data collection instrument. Participants were 366 all of them teachers of Secretaria de Estado da Educação do Distrito Federal. They worked in different schools and various regions of Distrito Federal. Linear and interaction relations were tested for hypothesis checking. The obtained results showed that emotional exhaustion and ethical leadership are linearly related to concentration at work. However, they do not relate to the loss of ability to complete the work. In the latter, leadership appears as an important moderating variable. High levels of emotional exhaustion tend to converge negatively on assessments of leadership and presenteism. This study contributes to the understanding of the relationship between well-being and productivity and its moderating variables, as well as highlights that emotional exhaustion at work influence the teacher's assessment.
\end{abstract}

Keywords: presenteism; burnout syndrome; ethical leadership.

As organizações se preocupam com o desempenho das empresas e dos trabalhadores (PAAUWE; WRIGHT; GUEST, 2013). Historicamente esse desempenho era mensurado de maneira dura: pelo absenteísmo (BARGAS; MONTEIRO, 2014) ou com medidas financeiras. Entretanto, essas medidas duras apresentam diversos problemas: um é que presença não é sinônimo de produtividade (PALHA, 2014); outro é que, para trabalhos em contextos públicos, tipicamente negligenciados na literatura, e em empresas em que o trabalho é fundamentalmente emocional ou de cuidado, como educação e saúde, elas fazem menos sentido (PAAUWE; WRIGHT; GUEST, 2013). Assim, as medidas de afastamento interpretadas como medidas de contraprodutividade (TARIS; SCHAUFELI, 2015) ou de perda de produtividade no trabalho (FERREIRA et al., 2010; TAVARES; KAMIMURA, 2012) podem auxiliar, pois podem ser mais nefastas do que o absenteísmo e às vezes com maior prevalência (FERREIRA et al., 2010, 2017; PASCHOALIN,

\footnotetext{
Endereço para correspondência: Secretaria de Economia, Finanças e Administração da Aeronáutica - SEFA. Esplanada dos Ministérios, Bloco M, $3^{\circ}$ andar. Brasília, DF - Brasil. CEP: 70045-900.E-mails: vivi anepinheiro@hotmail. com,amalia.perez@ceub.edu.br, Aristides.Ferreira@iscte-iul.pt, marina.kohlsdorf@ceub.edu.br

Os dados completos dos autores encontram-se ao final do artigo.
}

2012; TAVARES; KAMIMURA, 2012; UMANN; GUIDO; GRAZZIANO, 2012), ainda mais no contexto escolar (PÉREZ-NEBRA; QUEIROGA; OLIVEIRA, 2020).

Uma das formas mais clássicas de se referir às perdas de desempenho no trabalho é pelo presenteísmo, que pode ser entendido como a incapacidade do trabalhador de produzir devido a problemas relacionados à saúde, mas que não o levam a se ausentar do trabalho (TAVARES; KAMIMURA, 2012); em outras palavras, o trabalhador comparece ao trabalho, mas produz abaixo do seu normal (KOOPMAN et al., 2002) por problemas de saúde ou de concentração (UMANN; GUIDO; GRAZZIANO, 2012).

Este fenômeno resulta em um retrabalho, que implica maiores gastos e custos para a organização. Além disso, um problema de saúde não devidamente tratado pode ter consequências mais severas (FERREIRA, et al., 2010). $\mathrm{O}$ presenteísta pode custar mais caro à organização do que aquele que simplesmente faltou e se recuperou. Com a falta, não há produtividade em um dia de trabalho, ao passo que, quando o trabalhador produz vários dias abaixo de suas possibilidades, as perdas podem ser rela- 
tivamente maiores; e, no caso de trabalhos cognitivos, é difícil mensurar essas perdas de forma objetiva (KOOPMAN et al., 2002).

Conforme afirmam Ferreira et al. (2010), apesar do presenteísmo ser um tema relativamente recente, existem inúmeros instrumentos de medida. A Stanford Presenteeism Scale, desenvolvida por Koopman et al. (2002), traduzida e validada para a população portuguesa por Ferreira et al. (2010), é um dos instrumentos mais utilizados. Ela trata de uma medida que avalia perdas de produtividade laboral através de dois fatores distintos: capacidade de concentração (exemplo: devido ao meu problema de saúde, as dificuldades que normalmente fazem parte do meu trabalho foram mais complicadas de gerir) e perda de performance apesar do problema de saúde (exemplo: no trabalho, consegui concentrar-me na concretização dos meus objetivos, apesar do meu problema de saúde). Este último fator incide nas causas físicas de presenteísmo e corresponde à quantidade de trabalho realizado sob efeito do presenteísmo, portanto, da superação para completar a tarefa apesar do problema de saúde. Em outras palavras, o primeiro fator prende-se a aspectos psicológicos e corresponde à quantidade de concentração mobilizada para produzir quando existe um efeito de presenteísmo (FERREIRA et al., 2010). O segundo fator incide nas causas físicas de presenteísmo e corresponde à quantidade de trabalho perdido sob efeito das causas de presenteísmo (FERREIRA et al., 2010).

Os estudos sugerem que há variáveis físicas, pessoais, socioprofissionais ou ocupacionais, organizacionais e da tarefa (PASCHOALIN, 2012; PEREIRA, 2014; SILVA, 2012; UMANN, 2011), preditoras do presenteísmo. Entretanto, as variáveis psicológicas são pouco estudadas. No caso de professores, a síndrome psicológica de maior prevalência é o burnout (BOYD et al., 2015). A síndrome de burnout é um processo de adoecimento decorrente de um período prolongado de estresse profissional (FRANÇA, 2014; RIBEIRO; COSTA NETO, 2014) devido à tensão emocional constante (CODO; VASQUES-MENEZES, 1999), sendo esta dimensão a mais típica (CARLOTTO, 2014; MASLACH; JACKSON; LEITER, 1997; SILVEIRA, 2014; TAMAYO; TRÓCCOLI, 2002; VAN HORN et al., 2004).

Os estudos indicam que o presenteísmo pode ser um consequente da síndrome de burnout (FERREIRA; MARTINEZ, 2012; PAZ, 2009; VIEIRA et al., 2013). O burnout é uma consequência de esforço prolongado no local de trabalho quando os recursos estão escassos, e associa-se ao presenteísmo. Há evidências de que há um custo para o indivíduo, a exaustão emocional, associado à finalização de tarefas profissionais quando se sofre de algum tipo de condição médica, podendo levar ao presenteísmo. A qualidade dos serviços prestados e o nível de produção fatalmente serão afetados (FERREIRA; MARTINEZ, 2012).

A relação linear entre exaustão emocional e presenteísmo, apesar de natural, ou seja, o sofrimento leva a uma baixa de produção, relaciona-se à tese do "trabalhador feliz e produtivo" (STAW, 1986; PEIRÓ et al., 2019), neste caso, no quadrante, trabalhador infeliz-improdutivo (hi- pótese 1). Embora seja uma relação esperada, quais seriam possíveis variáveis moderadoras desta relação? Em outras palavras: o que poderia ser feito para amenizar o impacto do sofrimento no desempenho?

A literatura aponta que a liderança tem importante influência tanto no desempenho (hipótese 2) quanto no bem-estar das pessoas; assim, a liderança poderia ser um moderador desta relação (hipótese 3) (ALFAIATE; SANTOS, 2016; PISCO, 2015; NIEMEYER; CAVAZOTTE, 2016; JORGE, 2016; KALSHOVEN; BOON, 2012) e nos indivíduos. $\mathrm{O}$ estilo de liderança dos gestores, quando insatisfatório, é outro fator determinante para o aparecimento do burnout.

Brown, Treviño e Harrison (2005, p. 120) definem liderança ética como "a demonstração de conduta apropriada através de ações pessoais e relações interpessoais, bem como a promoção de tal conduta entre os seguidores através de uma comunicação bidirecional, do reforço e da tomada de decisão". Atribui-se ao líder ético grande influência no que concerne ao comportamento de seus seguidores.

O contexto deste trabalho foram escolas públicas. Vale notar que as escolas, apesar de estarem sob uma mesma política educacional, funcionam como pequenas e médias organizações. Brasília, hoje, contém 700 escolas públicas. Cada uma com sua liderança própria e bastante autônoma em termos de decisão. Vale ressaltar ainda que a exaustão emocional, variável mais típica do burnout, é uma variável emotiva com importante função nas avaliações dos indivíduos (EDWARDS, 1990; NIELSEN et al., 2017; PETTY; WEGENER, 1991). Portanto, espera-se que (hipótese 4) os níveis baixos de exaustão emocional apresentem maior variabilidade de interpretação e sejam mais influenciados pelo ambiente, portanto também pela liderança, quando comparados aos níveis mais altos, em que a avaliação tende a ser enviesada pela própria emoção. A Figura 1 descreve as relações esperadas e as hipóteses propostas neste estudo.

Figura 1: Proposta de modelo de pesquisa dos preditores de presenteísmo.

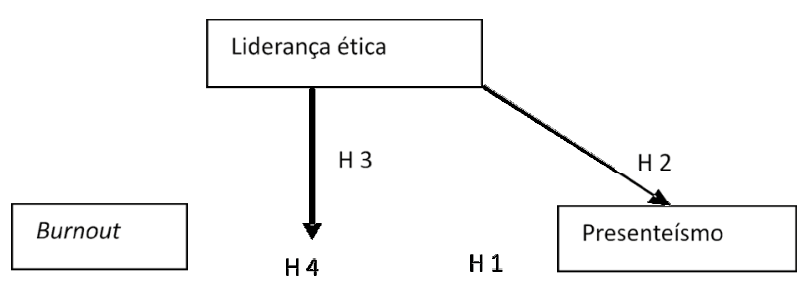

\section{Método/participantes}

Participaram da pesquisa 366 professores do ensino fundamental do Distrito Federal: Plano Piloto (165) e das cidades satélites da Candangolândia (23), Itapoã (14), Recanto da Emas (41), Riacho Fundo (45) e de Taguatinga (24). 
Dentre os pesquisados, a maioria era constituída por mulheres (73,95\%); 63,0\% eram casados. Em relação ao tipo de contrato de trabalho, $74,4 \%$ eram concursados. A média de idade da amostra foi de 41,07 anos ( $\mathrm{DP}=9,30$ ), com uma média de experiência profissional de 15,09 anos $(\mathrm{DP}=8,84)$ e uma média de trabalho na atual escola de 15,09 anos $(\mathrm{DP}=6,14)$. Estes dados são coerentes com os divulgados pela Secretaria de Educação sobre a sua distribuição censitária.

\section{Instrumentos}

Presenteismo. Mensurado pela escala Stanford Presenteeism Scale (SPS-6). A versão original deste instrumento foi desenvolvida e validada por Koopman e colaboradores (2002), e os primeiros trabalhos de tradução da SPS6 para a língua portuguesa foram realizados por Ferreira (FERREIRA et al., 2010; FRAUENDORF; PINHEIRO; CICONELLI, 2014). A SPS-6 é uma medida que avalia as perdas de produtividade laboral através de dois fatores distintos: concentração no trabalho (alfa $=0,82)$ e perda de capacidade para completar o trabalho (alfa $=0,74$ ).

Sindrome de Burnout. Mensurado pelo fator de exaustão emocional da escala Maslach Burnout Inventory - MBI. A versão original foi desenvolvida por Maslach, Jackson e Leiter (1997), e foi traduzida e adaptada para o português brasileiro por Tamayo (1997). A consistência interna das três dimensões do inventário é satisfatória. $\mathrm{O}$ valor do alfa foi de 0,90 para exaustão emocional, de 0,82 para baixa realização profissional e 0,54 para despersonalização.

Liderança Ética. Questionário composto pela Ethical Leadership Scale (ELS). A versão original foi desenvolvida por Brow, Treviño e Harrison (2005). A Escala de Liderança Ética utiliza dez itens, dos quais sete medem o comportamento de princípio e três, a relação interpessoal positiva, aqui considerada como constructo único: Liderança Ética. O alpha de Cronbach foi de 0,93.

\section{Procedimento de coleta de dados}

Apresentou-se aos professores o Termo de Consentimento Livre e Esclarecido - TCLE para leitura e assinatura, após esclarecimento das dúvidas. Realizava-se uma apresentação garantindo o sigilo dos dados e confidencialidade das informações no que se refere aos funcionários da referida organização com autorização pelo Comitê de Ética CAAE: 53743316.2.0000.0023. Logo que os professores respondiam aos questionários, era fei- to o recolhimento e o agradecimento. A secretaria atua nos programas de educação infantil, ensino fundamental e ensino médio no Distrito Federal, garantindo a conformidade das ações e dos processos de gestão educacional às diretrizes governamentais vigentes.

Foram distribuídos 500 questionários, e 312 retornaram válidos. A estratégia para realização da pesquisa foi redefinida, passando a ser realizada também por e-mail, o qual continha um link dando acesso ao questionário disponibilizado em um provedor de serviços de pesquisa on-line $(\mathrm{N}=54)$. A pesquisa ficou disponível no mês de julho de 2016, e a divulgação foi feita através de grupos de professores do DF em redes sociais. Com esta estratégia aumentou-se o número de respondentes, mas perdeu-se a possibilidade de identificação das escolas e de realizar análise multinível (agregando as percepções de liderança).

\section{Procedimento de análise}

As análises iniciais demonstraram dados relativamente normais para todas as escalas, com exceção de duas dimensões de burnout: Despersonalização e Realização Profissional. Em função da não normalidade deste dado e da impossibilidade de realizar análises de interação, estas dimensões não foram utilizadas nessas análises.

Para o alcance do objetivo deste trabalho realizaram-se análises exploratórias de correlação entre as variáveis. Testaram-se as dimensões conjuntas de presenteísmo e este não apresentou relações relevantes; de modo que tais dimensões foram desprezadas, utilizando-se apenas as dimensões separadas. Para o teste de hipótese, utilizou-se regressão padrão para testar as hipóteses 1 e 2 ; teste de mediação para testar a hipótese 3 ; e gráfico para testar a hipótese 4 .

\section{Resultados}

Resultados descritivos serão apresentados na seguinte sequência: presenteísmo, síndrome de burnout e liderança ética. $\mathrm{O}$ presenteísmo apresentou média de 3,30 $(\mathrm{DP}=1,06)$ para concentração no trabalho, e para perda de capacidade para completar o trabalho a média foi de $3,08(\mathrm{DP}=1,19)$. Em relação à síndrome de burnout, os professores pesquisados apresentaram valores médios de exaustão emocional $(\mathrm{M}=2,99 ; \mathrm{DP}=0,86)$. Na Escala de Liderança Ética a média foi de $3,87(\mathrm{DP}=0,80)$. Os dados resumidos encontram-se na Tabela 1.

Tabela 1: Análises descritivas de Presenteísmo, Burnout e Liderança Ética

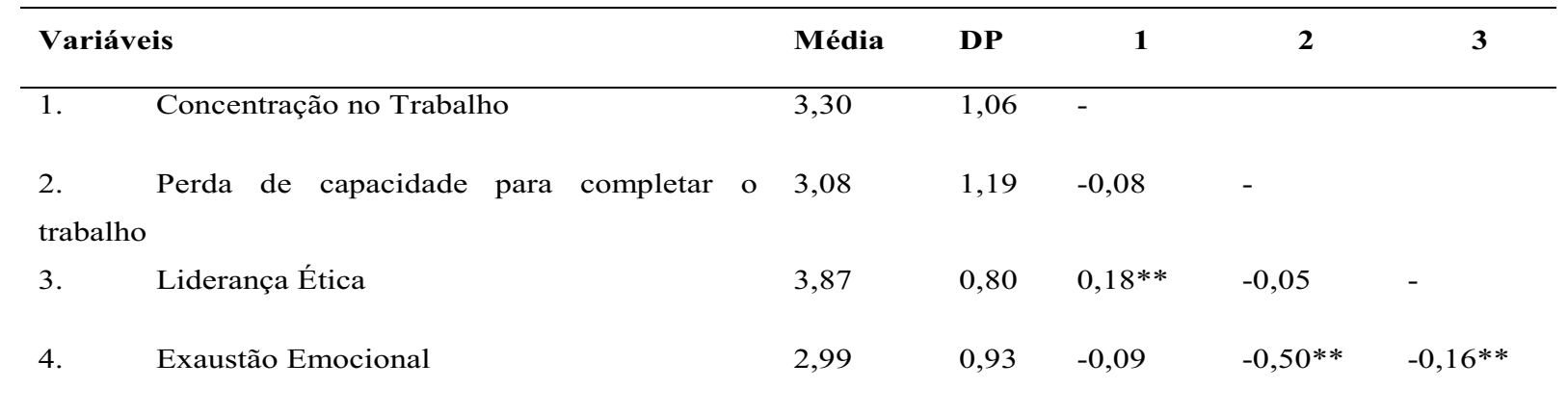

Notas. $* * p<.01 * p<.05$ 
Para testar as hipóteses em estudo, desenvolveu-se uma Regressão Linear Múltipla (Tabela 2), que permite predizer a capacidade de concentração dos professores quando estão doentes, bem como as perdas de capacidade para terminar as suas tarefas. Os resultados mostram que as variáveis liderança ética $(B=-.64, p<.01)$ e exaustão emocional $(B=-1.22, p<.01)$ surgem como preditores significativos de concentração no trabalho, e para perda de capacidade para completar o trabalho a predição é marginal em exaustão emocional e maior para liderança ética. Estas evidências suportam as hipóteses H1 e H2.
No que diz respeito à variável dependente perdas de capacidade para completar o trabalho, os dados sugerem que as variáveis liderança ética $(B=.69, p<.01)$ e exaustão emocional $(B=.52, p=.06)$ surgem como variáveis explicativas das perdas de capacidade dos professores com problema de saúde para terminarem os seus trabalhos. Tal como é possível verificar na Figura 3, constatamos que, em presença de líderes com maior liderança ética (+1DP) e situações de exaustão emocional, as perdas de capacidade para completar o trabalho são menos intensas $(B=-.20,95 \%$ CI [-.363, -.029], $t=-2.31, p<.01)$.

Tabela 2: Regressão linear dos preditores de concentração no trabalho e perdas de capacidade para completar o trabalho.

\begin{tabular}{|c|c|c|c|c|c|c|}
\hline & \multicolumn{3}{|c|}{ Concentração no trabalho } & \multicolumn{3}{|c|}{$\begin{array}{l}\text { Perda de capacidade para completar o } \\
\text { trabalho }\end{array}$} \\
\hline & \multirow[t]{2}{*}{$B$} & $S E$ & \multirow[t]{2}{*}{$t$} & \multirow[t]{2}{*}{$B$} & \multirow[t]{2}{*}{$S E B$} & \multirow[t]{2}{*}{$t$} \\
\hline & & $B$ & & & & \\
\hline Constante & 7.60 & .89 & $8.50^{* *}$ & .71 & .90 & .78 \\
\hline Liderança Ética & -.64 & .22 & $-2.88^{* *}$ & .69 & .22 & $3.06^{* *}$ \\
\hline Exaustão Emocional & -1.22 & .27 & $-4.47^{* *}$ & .52 & .28 & $1.87^{\dagger}$ \\
\hline Interação (Liderança Ética x Exaustão & .14 & .07 & $2.06^{*}$ & -.15 & .07 & $-2.18^{*}$ \\
\hline \multicolumn{7}{|l|}{ Emocional) } \\
\hline$R^{2}$ & \multicolumn{3}{|l|}{.29} & \multicolumn{3}{|l|}{.05} \\
\hline$F$ & \multicolumn{3}{|c|}{$42.83 * *$} & \multicolumn{3}{|c|}{$5.73 * *$} \\
\hline
\end{tabular}

Notas. $* * p<.01 * p<.05 \dagger p<.10$

A liderança ética interage com a exaustão emocional para explicar a concentração no trabalho $(B=.14, p<$ $.05)$ e perda de capacidade para completar o trabalho $(B$ $=-.15, p<.05)$, dando suporte para H3. A Figura 2 mostra-nos que em presença de líderes com maior liderança ética (+1DP) as perdas de concentração não são tão elevadas $(B=-.56,95 \%$ CI [-.721, -.392], $t=-6.64, p<.01)$ como em situações de baixa liderança ética (-1DP) ( $B$ $=-.79,95 \%$ CI $[-.951,-.627], t=-9.59, p<.01)$. Estas evidências suportam a H4.

Figura 2: Análise da interação entre a exaustão emocional e a liderança ética na explicação da concentração no trabalho.

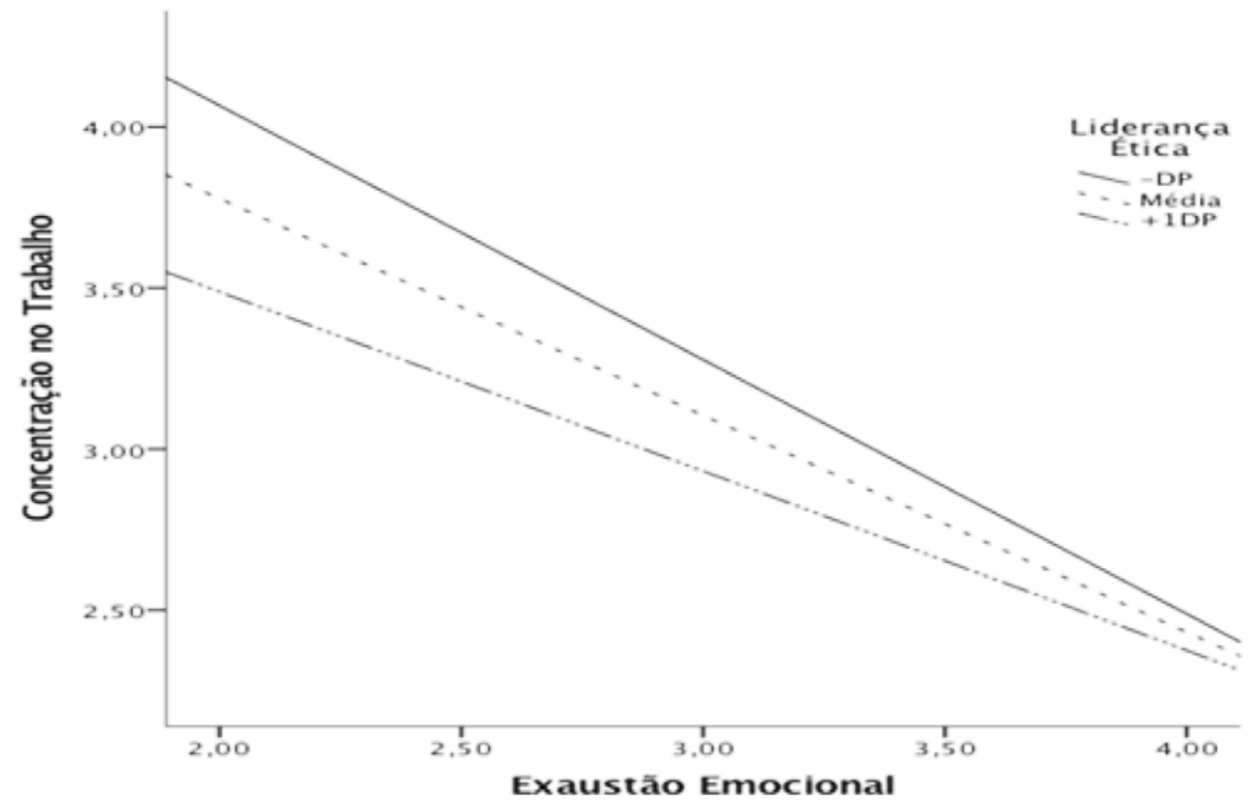


Figura 3: Análise da interação entre a exaustão emocional e a liderança ética na explicação das perdas de capacidade para completar o trabalho.

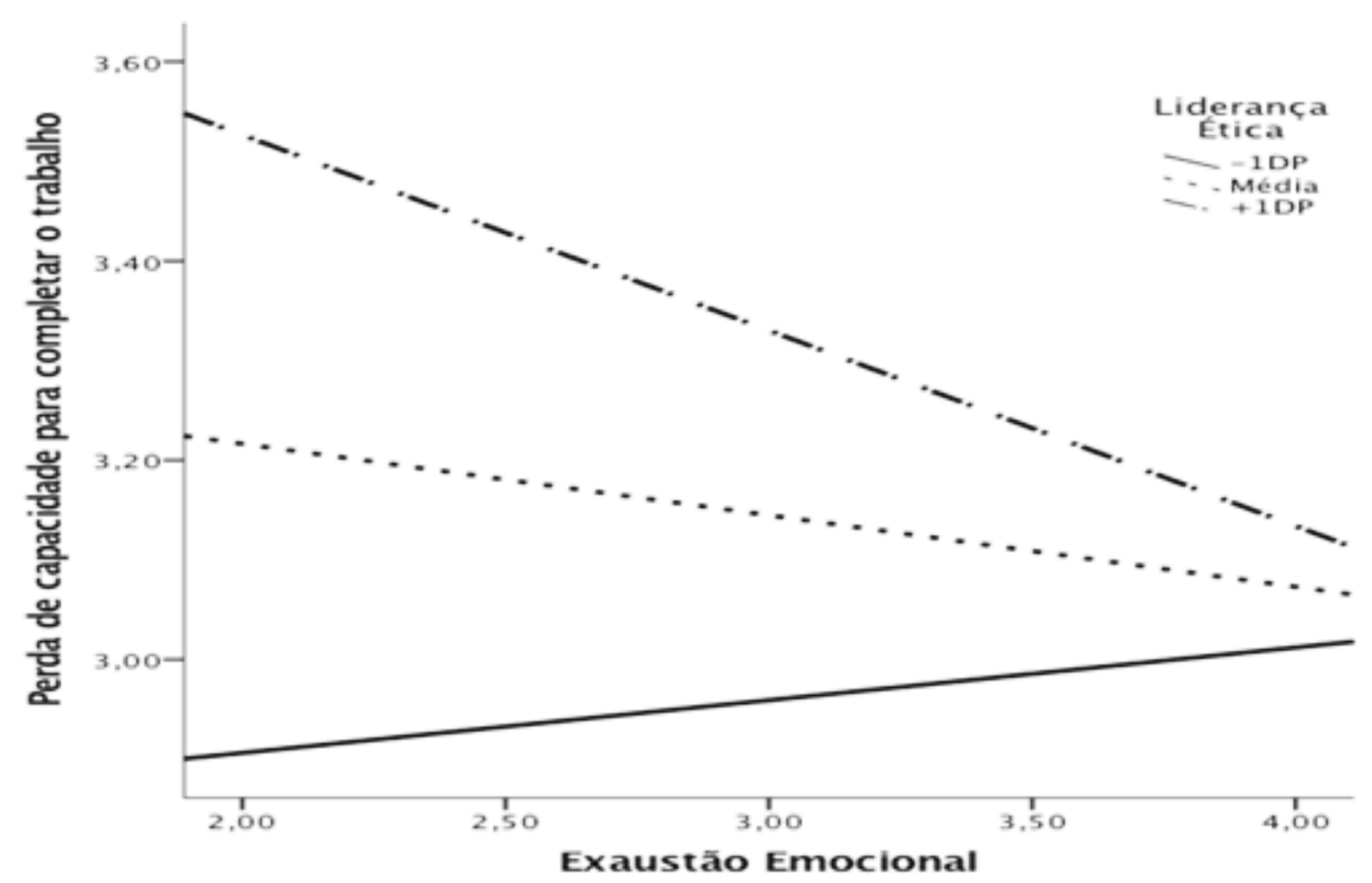

\section{Discussão}

Neste estudo, o objetivo foi identificar variáveis que podem explicar as perdas de desempenho no trabalho no contexto escolar. Os resultados encontrados convergem com o que já foi apresentado na literatura, tanto em termos de descrição quanto de proposta. Os dados relativos à exaustão emocional, dimensão mais típica do burnout, variam mais quando comparados às demais (FREITAS et al., 2016; VAN HORN et al., 2004) e apresentam-se mais úteis para entender o processo do burnout e, de forma mais ampla, o mal-estar docente.

Os resultados também convergem para a proposta de Peiró et al. (2014, 2015), de que o mal-estar e a produtividade parecem não ter uma relação linear e são dependentes de outras variáveis mediadoras ou antecedentes, como o caso da liderança ética. A relação com a concentração no trabalho parece ser mais direta, mas a perda de capacidade para completar o trabalho não é linear como se apresenta na regressão. Outro dado interessante é o fato de as emoções no trabalho (alta exaustão emocional) ter um efeito de conversão na interpretação tanto do presenteísmo quanto da liderança ética, como já foi apontado na literatura (EDWARDS, 1990; NIELSEN et al., 2017; PETTY; WEGENER, 1991). Resultados similares com exaustão emocional e desempenho também já foram relatados (DANIELS; HARRIS, 2000). Em outras palavras, quando a exaustão emocional é muito intensa, quase que a despeito do que se faça, o presenteísmo estará presente, independentemente do estilo de liderança. Do ponto de vista prático, isso implica a necessidade de trabalhar com o gerenciamento das emoções (BAUMEISTER et al., 2007) no caso de altos níveis de exaustão emocional, antes de intervir com outras variáveis no contexto organizacional.

Os resultados sugerem que o adoecimento do profissional, ou seja, a maior exaustão emocional, em geral, acarreta pior resultado tanto para a concentração profissional quanto para a capacidade de completar o trabalho. Embora os dados apresentem tendências esperadas (maior exaustão emocional, pior desempenho), alguns resultados são menos esperados com relação à perda de capacidade para completar o trabalho.

Um resultado interessante é que a liderança, no caso da concentração no trabalho, apresenta uma contribuição relativamente baixa, pois os grupos se diferenciam pouco. Entretanto, no caso da necessidade de superar a dificuldade para completar o trabalho, o desafio é que a menor liderança ética sugere maiores níveis de necessidade de superar esta dificuldade e, portanto, maior produtividade por parte do trabalhador. Este dado é desafiador, pois uma das leituras possíveis é que aparentemente o líder abusivo pode ser efetivo para aumentar a produção (TEPPER; SIMON; PARK, 2017); embora pareça lógico, ele pode ser também transitório, pois o que as pessoas farão em curto ou médio prazo é se abster do trabalho (VIEIRA, 2017). Outra explicação para este fenômeno é que o líder menos ético está relacionado com maior necessidade de habilidade para completar o trabalho; quando o líder é ético, essa necessidade diminui, portanto se completa a tarefa com menos presenteísmo. Finalmente o fenômeno pode ser explicado pela aceleração do trabalhador. A aceleração foi apresentada por Dejours em 1987 e tem sido descrita com frequência nas pesquisas sobre trabalho (e.g. BLANCH; OCHOA; CABALLE- 
RO, 2018). Ela sugere que, em um contexto problemático em termos de liderança, para proteger-se o indivíduo acelera seu comportamento tentando evitar uma piora de desempenho, mas gera custos em outras ordens.

\section{Conclusão}

Este trabalho contribui para a literatura de bem-estar sustentável quando consegue explicar e encontrar resultados tanto sinérgicos quanto antagônicos e esses resultados são coerentes. Especificamente se entende que o mal-estar, neste caso a alta exaustão emocional, pode gerar avaliações negativas, sejam elas sobre o contexto ou sobre o próprio desempenho. Ademais, as relações não lineares entre bem-estar e desempenho podem ser mediadas pela liderança para a completude de tarefas, mas para a concentração no trabalho não, sugerindo a necessidade de novos estudos nos quais seja possível explicar em que condições a relação entre bem-estar e produtividade pode se dar e em que casos ela é mediada ou predita por outras variáveis. Também contribui com a Secretaria de Educação por explicar parte da problemática encontrada no contexto educacional brasileiro, quando há uma interpretação divergente entre a prática do gestor e a do professor.

O trabalho também apresenta limitações quanto ao número de escolas estudadas, o que impossibilitou uma análise multinível, e ainda pelo fato de as variáveis serem de autorrelato e não haver inclusão de medidas de outras fontes, como índices IDEB das escolas. Sugere-se, para futuras pesquisas, descrever e entender uma liderança abusiva (ou antiética) nesse contexto, como por exemplo a liderança sombra (JORGE, 2016), além de operacionalizar o modelo com outras variáveis relativas ao bem-estar e produtividade e variáveis de contexto relativas ao desenho do trabalho. Entende-se que estas e outras questões podem ser a base para uma pesquisa de mais larga escala na área de educação.

\section{Informações sobre os autores:}

Viviane Pinheiro Lourenço

(iD) http://orcid.org/0000-0002-7486-9476

(9) http://lattes.cnpq.br/3923203342136007

Mestrado em Psicologia no Centro Universitário de Brasília/ UniCEUB. Especialista em Comportamento Organizacional e Gestão de Pessoas pela Escola Superior Aberta do Brasil - ESAB (2014). Especialista em Gestão e Docência do Ensino Superior. Licenciatura em Pedagogia (em andamento). Graduação em Administração pela Universidade Estadual Vale do Acaraú (2011). Pesquisou os seguintes temas: Adoecimento Docente, burnout, Absenteísmo, Presenteísmo, Estratégias de Coping, Liderança, Motivação Humana, Clima Organizacional, Gestão de Pessoas e Recursos Humanos. Experiência em rotinas administrativas em geral e recrutamento, seleção, cargos e salários, treinamento, avaliação de desempenho, administração de clima. Monitora a disciplina de Recursos Humanos. Parecerista em bancas de graduação. Atualmente trabalha como Coordenadora Acadêmica da Faculdade Laboro - Unidade Brasília.
Amalia Raquel Pérez-Nebra

(iD) http://orcid.org/0000-0001-8386-1233

\section{(9) http://lattes.cnpq.br/4432860581877711}

É professora titular do Centro Universitário de Brasília; membro da Sociedade Brasileira de Psicologia Organizacional e do Trabalho (SBPOT), da Associação Nacional de Pesquisa em Psicologia (ANPEPP) e da Associação Internacional de Psicologia Aplicada (IAAP). Coordena projetos de psicologia social aplicada em diferentes contextos: Tribunal de Justiça do Distrito Federal e Território (econômico), Hospital Materno-Infantil de Brasília (trabalho) e outros projetos de investigação em psicologia social. É professora do Centro Universitário de Brasília (UniCEUB) desde 2005. Atua na graduação da Comunicação Social (psicologia social aplicada entre 2005-2010) e da Psicologia (2010 - atual). $\mathrm{Na}$ psicologia leciona Práticas em Psicologia Social, Teorias em Psicologia Social, Psicologia Organizacional, Psicologia do Trabalho, Psicologia Positiva, metodologia de pesquisa, estatística e orientação de trabalhos individuais. É filiada ao Programa de Pós-graduação em Psicologia, na linha de pesquisa de saúde, onde orienta mestrados, leciona e desenvolve pesquisas na área de saúde do trabalho. Fez doutorado sanduíche na Universidad de Madrid e obteve licença sabática na Universitat de València.

\section{Aristides Isidoro Ferreira}

\section{(iD) https://orcid.org/0000-0002-8280-1623}

\section{(9) https://ciencia.iscte-iul.pt/authors/aristides-i-ferreira/cv}

Assistant Professor at ISCTE - Instituto Universitário de Lisboa, Portugal, where he is the Director of the Master program in Human Resource Management and Organizational Consulting. He is also a researcher at the Business Research Unit in the same institute. He earned his $\mathrm{PhD}$ in Psychology from University of Minho, Portugal and he worked as a researcher for the Freie Universita" t Berlin, Germany. His research interests include psychological assessment, leadership, change management and presenteeism. His research work has appeared in journals such as The International Journal of Project Management, Journal of Business Research, International Journal of Human Resource Management, and Stress and Health.

\section{Marina Kohlsdorf \\ (iD) http://orcid.org/0000-0002-7029-3270 \\ (3) http://lattes.cnpq.br/2498980760258215}

Doutora e mestre em Processos de Desenvolvimento Humano e Saúde pelo Programa de Pós-Graduação em Processos de Desenvolvimento Humano e Saúde, no Instituto de Psicologia da Universidade de Brasília. Graduação em Psicologia (habilitações bacharel e psicóloga) pela Universidade de Brasília. Psicóloga na Unidade de Transplantes do Hospital Universitário de Brasília (HUB) de 2008 a 2012. Coordenadora adjunta do Curso de Especialização em Psicologia da Saúde (Instituto Brasiliense de Análise do Comportamento - IBAC) de 2010 a 2014. Docente de 2011 a 2013 no curso de Psicologia da Universidade Católica de Brasília - UCB. Professora no curso de Psicologia do Centro Universitário UniCeub desde 2013, nos cursos de graduação e mestrado em Psicologia, e membro do Comitê de Análise de Projetos de Pesquisa na mesma instituição. Coordenadora do curso de Mestrado Stricto Sensu em Psicologia no Centro Universitário UniCeub desde 2019. Psicóloga na Secretaria de Saúde do Distrito Federal no Hospital Materno-Infantil de Brasília (HMIB) desde 2013. Colaboradora no Laboratório de Desenvolvimento em Condições Adversas - LADVERSA - na Universidade de Brasília (UnB). Editora associada da revista Psicologia: Reflexão e Crítica. Membro do Grupo de Trabalho da ANPEPP sobre Psicologia da Saúde da Criança e do Adolescente.

\section{Contribuições dos autores:}

Todos os autores colaboraram ao longo do processo, desde a elaboração até a revisão final do manuscrito. Os autores aprovaram o manuscrito final para publicação. 


\section{Como citar este artigo:}

ABNT

LOURENÇO, Viviane Pinheiro et al. Relação entre presenteísmo, síndrome de burnout e liderança ética em organizações escolares. Fractal: Revista de Psicologia, Niterói, v. 32, n. esp., p. 218-226, jun. 2020. https://doi.org/10.22409/1984-0292/v32_i-esp/40568

APA

Lourenço, V. P., Pérez-Nebra, A. R., Ferreira, A. I., \& Kohlsdorf, M. (2020, Junho). Relação entre presenteísmo, síndrome de burnout e liderança ética em organizações escolares. Fractal: Revista de Psicologia, 32(esp.), 218-226. doi: https://doi.org/10.22409/19840292/v32_i-esp/40568

\section{Copyright:}

Copyright (C) 2020 Lourenço, V. P., Pérez-Nebra, A. R., Ferreira, A. I., \& Kohlsdorf, M. Este é um artigo em acesso aberto distribuído nos termos da Licença Creative Commons Atribuição que permite o uso irrestrito, a distribuição e reprodução em qualquer meio desde que o artigo original seja devidamente citado.

Copyright (C) 2020 Lourenço, V. P., Pérez-Nebra, A. R., Ferreira, A. I., \& Kohlsdorf, M. This is an Open Access article distributed under the terms of the Creative Commons Attribution License, which permits unrestricted use, distribution, and reproduction in any medium, provided the original article is properly cited.

\section{Referências}

ALFAIATE, Helena Sofia; SANTOS, Joana Vieira dos. Liderança ética e marketing interno: análise das repercussões na satisfação e empenhamento de profissionais ativos. Revista E-Psi, v. 6, n. 2, p. 26-44, 2016. Disponível em: https:// revistaepsi.com/artigo/2016-ano6-volume2-artigo2/. Acesso em: 12 out. 2019

BARGAS, Eliete Boaventura; MONTEIRO, Maria Inês. Fatores relacionados ao absenteísmo por doença entre trabalhadores de Enfermagem. Acta Paulista de Enfermagem, São Paulo, v. 27, n. 6, p. 533-538, 2014. https://doi.org/10.1590/19820194201400087

BAUMEISTER, Roy et al. How emotion shapes behavior: feedback, anticipation, and reflection, rather than direct causation. Personality and social psychology review, v. 11, n. 2, p. 167-203, 2007. https://doi.org/10.1177/1088868307301033

BLANCH, Josep Maria; OCHOA, Paola; CABALLERO, Maria Fernanda. Over engagement, protective or risk factor of Burnout? In: DURBANO, F. (Ed.). Sustainable Management Practices. London: IntechOpen, 2018. https://doi.org/10.5772/ intechopen. 81746

BOYD, Carolyn et al. Emotion work in service occupations: link to well-being and job performance. In: VELDHOVEN, M. Van; PECCEI, R. (Ed.). Well-being and performance at work. New York: Psychology Press, 2015. p. 53-74.

BROWN, Michael; TREVIÑO, Linda; HARRISON, David. Ethical leadership: a social learning perspective for construct development and testing. Organizational behavior and human decision processes, v. 97, n. 2, p. 117-134, 2005. https://doi. org/10.1016/j.obhdp.2005.03.002

CARLOTTO, Mary Sandra et al. Burnout em professores: diferença e análise de gênero. Contextos clínicos, v. 7, n. 1, p. 86-93, 2014. Disponível em: http://pepsic.bvsalud.org/pdf/ cclin/v7n1/v7n1a09.pdf. Acesso em: 21 jan. 2018.
CODO, Wanderley; VASQUES-MENEZES, Ione (Org.). O que é burnout? In: _. Educação: carinho e trabalho. Rio de Janeiro: Vozes, 1999. p. 237-255.

DANIELS, Kevin; HARRIS, C. Work, psychological wellbeing and performance. Occupational Medicine, v. 50, n. 5, p. 304-309, 2000. https://doi.org/10.1093/occmed/50.5.304

EDWARDS, Kari. The interplay of affect and cognition in attitude formation and change. Journal of Personality and Social Psychology, v. 59, n. 2, p. 202-216, 1990. https://doi. org/10.1037/0022-3514.59.2.202

FERREIRA, Aristides Isidoro et al. Tradução e validação para a língua portuguesa das escalas de presentismo WLQ-8 E SPS-6. Avaliação Psicológica, Porto Alegre, v. 9, n. 2, p. 253266, 2010. Disponível em: http://pepsic.bvsalud.org/scielo. php?script=sci_arttext\&pid=S1677-04712010000200010\&lng $=$ pt\&nrm=iso. Acesso em: 11 mar. 2019.

FERREIRA, Aristides Isidoro et al. Working sick and out of sorts: a cross-cultural approach on presenteeism climate, organizational justice and work-family conflict. The International Journal of Human Resource Management, v. 30, n. 19, p. 2754-2776, 2017. https://doi.org/10.1080/09585192.2 017.1332673

FERREIRA, Aristides Isidoro; MARTINEZ, Luis. Presenteeism and burnout among teachers in public and private portuguese elementary schools. The International Journal of Human Resource Management, v. 23, n. 20, p. 4380-4390, 2012. https://www.tandfonline.com/doi/full/10.1080/09585192.2012 .667435

FRANÇA, Thaís Lorena Barbosa de et al. Burnout syndrome: characteristics, diagnosis, risk factors and prevention. Journal of Nursing, v. 8, n. 10, 2014, p. 3539-3546. Disponível em: https://periodicos.ufpe.br/revistas/revistaenfermagem/article/ view/10087. Acesso em: 30 ago. 2019.

FRAUENDORF, Renata; PINHEIRO, Marcelo de Medeiros; CICONELLI, Rozana Mesquita. Translation into Brazilian Portuguese, cross-cultural adaptation and validation of the Stanford presenteeism scale- 6 and work instability scale for ankylosing spondylitis. Clinical Rheumatology, v. 33, n. 12, p. 1751-1757, 2014. https://doi.org/10.1007/s10067-013-2429-6

FREITAS, Clarissa Pinto Pizarro et al. Impact of Job-Related Well-Being on the Relationship of Self-Efficacy With Burnout. Paidéia, Ribeirão Preto, v. 26, n. 3, p. 45-52, 2016. https://doi. org/10.1590/1982-43272663201606

JORGE, Cátia Sofia Nunes. A importância da liderança ética nas organizações: um estudo sobre a influência do narcisismo e do "efeito sombra" no comportamento ético do líder. 2016. Dissertação (Mestrado em Psicologia Social e das Organizações) - Instituto Universitário de Lisboa, Lisboa, 2016.

KALSHOVEN, Karianne; BOON, Corine. Ethical leadership, employee well-being, and helping the moderating role of human resource management. Journal of Personnel Psychology, v.11, n. 1, p. 60-68, 2012. https://doi.org/10.1027/1866-5888/ a000056

KOOPMAN, Cheryl et al. Stanford presenteeism scale: health status and employee productivity. Journal of Occupational and Environmental Medicine, v. 44, n. 1, p. 14-20, 2002. 
MASLACH, Christina; JACKSON, Susan E.; LEITER, Michael P. The Maslach Burnout Inventory Manual. In: ZALAQUETT, C. P.; WOOD, R. J. (Ed.). Evaluating stress: a book of resources. 3. ed. London: The Scarecrow Press, 1997. p. 191-218. Disponível em: https://smlr.rutgers.edu/sites/ default/files/documents/faculty_staff_docs/EvaluatingStress. pdf. Acesso em: 13 jan. 2020.

NIELSEN, Karina et al. Workplace resources to improve both employee well-being and performance: a systematic review and meta-analysis. Work and stress: an international journal of work, health \& organisations, v. 31, n. 2, p. 101-120, 2017. https://doi.org/10.1080/02678373.2017.1304463

NIEMEYER, Jeane Rodrigues Lucena; CAVAZOTTE, Flávia de Souza Costa Neves. Liderança ética, relacionamento líder-seguidor e desempenho: um estudo em uma empresa de telecomunicações. RAM - Revista de Administração Mackenzie, São Paulo, v. 17, n. 2, p. 67-92, 2016. https://doi. org/10.1590/1678-69712016/administracao.v17n2p67-92

PAAUWE, Jaap; WRIGHT, Patrick; GUEST, David (Ed.). HRM and performance: what do we know and where should we go? In: _. Human resource management and

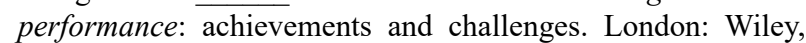
2013. p. 1-13.

PALHA, Cátia Daniela. O presentismo em enfermagem. 2014. Dissertação (Mestrado) - Escola Superior de Enfermagem do Porto, Porto, Portugal, 2014.

PASCHOALIN, Heloisa Campos. Presente no trabalho, mesmo doente: o presenteísmo na enfermagem. 2012. Tese (Doutorado) - Escola de Enfermagem Anna Nery, Universidade Federal do Rio de Janeiro, Rio de Janeiro, 2012.

PAZ, Mayara Pereira. O estresse no cotidiano de professores da educação superior. Revista FSA (Centro Univeristário Santo Agostinho), Teresina, v. 6, n. 1, 2009. Disponível em: http:// www4.fsanet.com.br/revista/index.php/fsa/article/view/425. Acesso em: 17 out. 2018.

PEIRÓ, José Maria et al. Bienestar sostenible en el trabajo: revisón y reformulación. Papeles del Psicólogo, v. 35, n. 1, p. 5-14, 2014. Disponível em: http://www.papelesdelpsicologo. es/pdf/2316.pdf. Acesso em: 23 mar. 2019.

PEIRÓ, José Maria et al. Bienestar sostenible en el trabajo. Conceptualización, antecedentes y retos. Psiencia: Revista Latinoamericana de Ciencia Psicológica, v. 7, n. 1, p. 133-135, 2015. https://doi.org/10.5872/psiencia/7.1.0116

PEIRÓ, José Maria et al. Longitudinal well-being and performance cross-cultural relation: a comparison between Brazil, Equator and Italy. In: ANSEEL, F. (Ed.). Working for the greater good: inspiring people, designing jobs and leading organizations for a more inclusive society. Turin: EAWOP - European Association of Work and Organizational Psychology, 2019. p. 1443.

PEREIRA, Nathália Coimbra. Presenteísmo odontológico: conhecendo um instrumento de pesquisa para mensuração e avaliação. 2014. Dissertação (Mestrado) - Programa de Pósgraduação em Odontologia da Universidade de São Paulo, São Paulo, 2014.

PÉREZ-NEBRA, Amália; QUEIROGA, Fabiana; OLIVEIRA, Thiago Aguiar. Presenteísmo de professores regentes: bem-estar como estado psicológico crítico na mediação de características do trabalho. Revista de Administração do Mackenzie, São Paulo, v. 21, n. 1, p. 1-27, 2020. https://doi:10.1590/1678-6971/ eRAMD200123

Fractal, Rev. Psicol., v. 32 - n. esp., p. 218-226, 2020
PETTY, Richard; WEGENER, Duane. Thought systems, argument quality, and persuasion. In: WYER, R. S.; SRULL, T. K. (Ed.). Advances in social cognition. Hillsdale: Erlbaum, 1991. v. 4, p. 147-161.

PISCO, Márcia Lúcia Roques. A vantagem da liderança no feminino: será que a perceção que as pessoas têm sobre a eficácia da liderança difere consoante o género? 2015. Dissertação (Mestrado em Psicologia) - Universidade de Évora, Portugal, 2015.

RIBEIRO, Keila Maria Moura Silva; COSTA NETO, Sebastião Benício da. Síndrome de burnout e estratégias de enfrentamento psicológico de professoras de programas strito sensu de uma universidade no Centro-Oeste brasileiro. Integración Académica en Psicología, v. 2, n. 4, p. 131-148, 2014.

SILVEIRA, Kelly Ambrosio et al. Estresse e enfrentamento em professores: uma análise da literatura. Educação em Revista, Belo Horizonte, v. 30, n. 4, p. 15-36, 2014. https://doi. org/10.1590/S0102-46982014000400002

SILVA, Andrea Martiniano da. Aplicação de técnica da mineração de dados na identificação do perfil de empregados absenteístas e presenteístas em uma empresa de courier da cidade de São Paulo. 2012. 130 f. Dissertação (Mestrado) Universidade Nove de Julho - UNINOVE, São Paulo, 2012.

STAW, Barry. M. Organizational psychology and the pursuit of the happy/productive worker. California Management Review, v. 28, n. 4, p. 40-53, 1986. https://doi.org/10.2307/41165214

TAMAYO, Mauricio Robayo. Relação entre a Síndrome de burnout e os valores organizacionais no pessoal de enfermagem de dois hospitais públicos. 1997. 123f. Dissertação (Mestrado) - Programa de Pós-graduação em Psicologia, Universidade de Brasília, DF, 1997.

TAMAYO, Maurício Robayo; TRÓCCOLI, Bartholomeu Tôrres. Exaustão emocional: relações com a percepção de suporte organizacional e com as estratégias de coping no trabalho. Estudos de Psicologia, Natal, v. 7, n. 1, p. 37-46, 2002. https://doi.org/10.1590/S1413-294X2002000100005

TARIS, Toon; SCHAUFELI, Wilmar. Individual well-being and performance at work: a conceptual and theoretical overview. In: VELDHOVEN, M. van; PECCEI, R. (Ed.). Well-being and performance at work. New York: Psychology Press, 2015. p. 15-34.

TAVARES, Renata; KAMIMURA, Quésia Postigo. Gestão em saúde corporativa: absenteísmo e presenteísmo. In: INTERNATIONAL CONGRESS UNIVERSITY INDUSTRY COOPERATION, 4., 2012, Taubaté. Disponível em: http:// www.unitau.br/unindu/artigos/pdf337.pdf. Acesso em: 13 nov. 2018.

TEPPER, Bennet J.; SIMON, Lauren; PARK, Hee Man. Abusive Supervision. Annual Review of Organizational Psychology and Organizational Behavior, v. 4, n. 1, p. 123-152, 2017. https:// doi.org/10.1146/annurev-orgpsych-041015-062539

UMANN, Juliane. Estresse, coping e presenteísmo em enfermeiros hospitalares. 2011. 132f. Tese (Mestrado) Programa de Pós-graduação em Enfermagem, Universidade Federal de Santa Maria, RS, 2011.

UMANN, Juliane; GUIDO, Laura de Azevedo; GRAZZIANO, Eliane da Silva. Presenteísmo em enfermeiros hospitalares. Revista Latino-Americana de Enfermagem, Ribeirão Preto, v. 20 , n. 1, p. 159-166, 2012. https://doi.org/10.1590/S010411692012000100021 
VAN HORN, Joan et al. The structure of occupational wellbeing: a study among Dutch teachers. Journal of Occupational and Organizational Psychology, v. 77, n. 3, p. 365-375, 2004. https://doi.org/10.1348/0963179041752718

VIEIRA, Lenamar Fiorese et al. Análise da síndrome de burnout e das estratégias de coping em atletas brasileiros de vôlei de praia. Revista Brasileira de Educação Física e Esporte, São Paulo, v. 27, n. 2, p. 269-276, 2013. https://doi.org/10.1590/ S1807-55092013005000012

VIEIRA, Lilian da Silva. Bem-estar no trabalho e afastamento dos profissionais da educação pública do Distrito Federal. 2017. Dissertação (Mestrado em Psicologia) - Centro Universitário de Brasília, Brasília, 2017. 